\title{
Capturing metabolic dynamics in mitochondria
}

\author{
A rapid method for isolating mitochon- \\ dria with exquisitely high purity helps \\ researchers profile metabolic dynamics \\ within this organelle. \\ Metabolites, the products of biochemi-
} cal reactions in the cell, have crucial roles in mammalian physiology. However, these tiny molecules are hard to pin down. Part of the problem is that the chemical structures of biological small molecules are extremely diverse. Another major challenge is that their levels in the cell can change rapidly depending on biological conditions. Both present hurdles for the accurate detection, identification and quantification of metabolites using mass spectrometry technology.

Researchers in David Sabatini's lab at the Whitehead Institute at the Massachusetts Institute of Technology have been interested in studying how organelles such as mitochondria communicate with other parts of the cell. Most metabolite profiling studies are performed with whole cells, but the researchers realized early on that they would need an approach to cleanly purify mitochondria to have the resolution needed to study the distinct metabolite profiles, or metabolomes, of these organelles under different cellular states.

Sabatini, first author Walter Chen and their colleagues considered using one of several established protocols for purifying mitochondria, but all had severe limitations when it came to profiling metabolites. "The problem with purification methods is that they are so slow; they take hours to get anything pure," says Sabatini. It was important that they use a rapid purification method because any residual enzyme activity could have substantial effects on the metabolome.

The team was willing to sacrifice yield for the sake of a rapid and specific method that resulted in pure mitochondrial preparations. They designed an immunopurification approach that required fusing a portion of the outer mitochondrial membrane protein OMP25 to three hemagglutinin (HA)

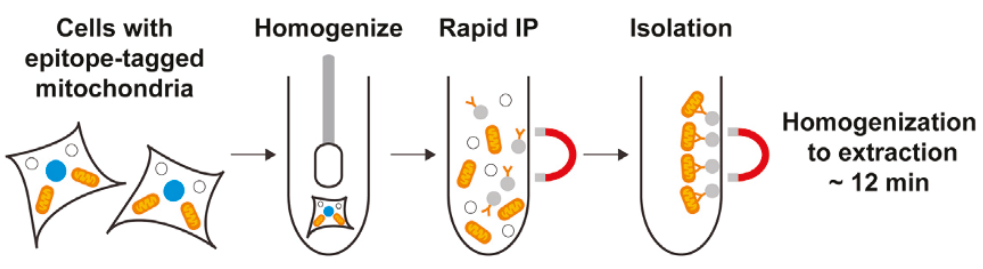

A rapid method for purifying mitochondria from cells enables mitochondrial metabolite profiling. IP, immunoprecipitation. Figure reprinted from Chen et al. (2016) with permission from Elsevier.

epitope tags. This allowed them to use antiHA beads to specifically capture mitochondria from cells broken open by mechanical grinding (homogenization).

Little tweaks to the purification method made a big difference, the researchers found. They originally started with agarose beads, as are routinely used in protein purification, but they soon discovered that metabolites were getting trapped inside the porous agarose material and throwing off their measurements. They found success by switching to smaller, impermeable magnetic beads. They also realized that the choice of purification buffer was crucial. "Typically, when people do mass spectrometry metabolite profiling of cells, they wash the cells and directly lyse the cells in, for example, a methanol-water buffer," says Sabatini. This process completely destroys the cells, so his team could not apply the approach to capture mitochondria intact. Other buffers commonly used for mitochondrial isolation were not appropriate, either, as they contain components that interfere with mass spectrometry analysis. In the end, they settled on a potassium-chloride-based buffer that was friendly both to mitochondria and to the mass spectrometer.

All together, these optimizations to develop a 12-minute method for mitochondrial metabolite extraction took the team a good year and a half to perfect, Sabatini recalls. Once the method was working well, they applied it to measure the mitochondrial and whole-cell concentrations of about 100 metabolites in HeLa cells predicted to be involved in respiratory chain function. In particular, they monitored the metabolic response to three different inhibitors used to treat mitochondrial disease. "If you look at the whole cell basis and you use a series of specific mitochondrial inhibitors that act on different parts of the [respiratory] chain, you really can't distinguish [the inhibitors] apart in terms of the metabolite effects they have," explains Sabatini. "But when you look in the mitochondria, [the effects are] dramatically different; there are very specific changes." He notes that the data imply that mutations impairing different components in the respiratory chain could have different clinical implications in people with mitochondrial disease.

The purification approach the researchers report may have an impact beyond the study of mitochondria. "We have it working beautifully for lysosomes," Sabatini says, noting that it should be adaptable to other organelles as well. He also suggests that the purification method could be useful for analyzing other mitochondrial content, such as DNA, RNA and proteins.

Sabatini reflects that their study was limited in scope by the need to build standard curves using authentic compounds in order to quantify the metabolites of interest. $\mathrm{He}$ looks forward to seeing more methodological advances in the field of metabolomics, which will enable researchers to discover novel metabolites in their samples.

\section{Allison Doerr}

\section{RESEARCH PAPERS}

Chen, W.W. et al. Absolute quantification of matrix metabolites reveals the dynamics of mitochondrial metabolism. Cell 166, 1324-1337 (2016). 